\title{
RESEARCH
}

Open Access

\section{Probucol enhances the therapeutic efficiency of mesenchymal stem cells in the treatment of erectile dysfunction in diabetic rats by prolonging their survival time via Nrf2 pathway}

Haoran Wang ${ }^{1 \dagger}$, Keqin Zhang ${ }^{1,2 \dagger}$, Zheng Ruan ${ }^{1,3}$, Dingqi Sun ${ }^{1,2}$, Hui Zhang ${ }^{1,2}$, Guiting Lin ${ }^{4}$, Liangliang Hu ${ }^{5}$, Shengtian $\mathrm{Zhao}^{1,2^{*}}$ and Qiang $\mathrm{Fu}^{1,2^{*}}$ (D)

\begin{abstract}
Background: Intracavernous injection of mesenchymal stem cells (MSCs) is a promising method for diabetic mellitus-induced erectile dysfunction (DMED), but short survival time of MSCs in cavernous is a fatal defect for therapy. This study investigated therapeutic efficiency and potential mechanism of probucol combined with MSCs.

Methods: In vivo study, a total of forty-eight 10-week-old male Sprague-Dawley (SD) rats were used. Twelve rats received intraperitoneal injection of PBS as the sham group; the rest received intraperitoneal injection of $60 \mathrm{mg} / \mathrm{kg}$ streptozotocin to establish DM models. DM rats were randomly divided into three groups: received intracavernosal (IC) injection of either PBS (DM group), MSCs (M group), or administrated probucol after intracavernosal injection of MSCs ( $P+M$ group). Erectile function was assessed by electrical stimulation of the cavernous nerves with real-time intracavernous pressure measurement. After euthanasia, penile tissue was investigated for histologic examination and Western blotting. In in vitro experiment, $\mathrm{H}_{2} \mathrm{O}_{2}$ was used to create oxidative stress environment to detect changes in cell viability. CCK8 was used to measure cell viability of MSCs treated with or without probucol. Intracellular ROS changes were detected by flow cytometry. Autophagy and apoptosis were detected by Western blotting and confocal microscopy.

(Continued on next page)
\end{abstract}

\footnotetext{
* Correspondence: zhaost2017@126.com; qiangfu68@126.com

${ }^{\dagger}$ Haoran Wang and Keqin Zhang contributed equally to this work

'Department of Urology, Shandong Provincial Hospital, Cheeloo College of

Medicine, Shandong University, Jingwuweiqi Road 324\#, Jinan 250021,

Shandong, People's Republic of China

Full list of author information is available at the end of the article
}

(c) The Author(s). 2020 Open Access This article is licensed under a Creative Commons Attribution 4.0 International License, which permits use, sharing, adaptation, distribution and reproduction in any medium or format, as long as you give appropriate credit to the original author(s) and the source, provide a link to the Creative Commons licence, and indicate if changes were made. The images or other third party material in this article are included in the article's Creative Commons licence, unless indicated otherwise in a credit line to the material. If material is not included in the article's Creative Commons licence and your intended use is not permitted by statutory regulation or exceeds the permitted use, you will need to obtain permission directly from the copyright holder. To view a copy of this licence, visit http://creativecommons.org/licenses/by/4.0/ The Creative Commons Public Domain Dedication waiver (http://creativecommons.org/publicdomain/zero/1.0/) applies to the data made available in this article, unless otherwise stated in a credit line to the data. 


\begin{abstract}
(Continued from previous page)
Results: Recovery of erectile function was observed in the P $+\mathrm{M}$ group. The combination therapy decreased fibrosis and increased endothelial function compared with MSC therapy alone. Western blotting results confirmed the increased expression of $\mathrm{Nrf} 2$ and $\mathrm{HO}-1$ in cavernous body. $\mathrm{H}_{2} \mathrm{O}_{2}$ induced high oxidative stress and reduced cell viability in vitro, which was gradually reversed with increased concentration of probucol. $\mathrm{H}_{2} \mathrm{O}_{2}$ reduced $\mathrm{Nrf} 2$ expression, which was reversed by probucol's intervention. Furthermore, the expression of Bax, Caspase3, and Cleaved-Caspase 3 decreased, and the expression of $\mathrm{BCl}-2$ increased in a dose-dependent manner because of probucol's intervention. In addition, Beclin1 and LC3II both increased in a dose-dependent manner. Meanwhile, the expression of P62 decreased. In the study of autophagy flux, we found probucol did not block it.
\end{abstract}

Conclusion: Probucol enhanced therapeutic efficiency of MSCs in DMED by prolonging their survival time, which mediated through improving the transplanted microenvironment of MSCs, increasing self-antioxidant ability of MSCs, strengthening protective autophagy, and inhibiting apoptosis of MSCs via Nrf2 pathway.

Keywords: Mesenchymal stem cell, Probucol, Oxidative stress, Nrf2, Autophagy, Apoptosis

\section{Background}

Erectile dysfunction (ED) is the consistent or recurrent inability to achieve and/or maintain a penile erection sufficient for satisfactory sexual performance [1]. Over the past few years, erectile dysfunction (ED) is a common complication in patients with diabetes mellitus (DM) [2]. Up to now, the mechanism of DMED is not fully understood. Increasing pieces of evidence show that oxidative stress is considered a major factor in the promotion and development of DMED [3]. Our previous studies confirmed that the DMED could be improved by attenuating oxidative stress.

At present, phosphodiesterase type 5 inhibitors (PDE5i) are the first line treatment for ED. But its effect on DMED is disappointing $[4,5]$. Intracavernous injection of mesenchymal stem cells (MSCs) is a novel therapy. It has been proved that intracavernous injection of MSCs could significantly improve DMED in preclinical research and human clinical trials $[6,7]$. The fatal defect of MSC therapy is the short maintenance time of curative effect, which is due to the short survival time of MSCs in the corpus cavernosum of the penis. In the corpus cavernosum of diabetic rats, the increase of reactive oxygen species (ROS) inhibits MSC proliferation, increases senescence, decreases differentiation, and inhibits MSC immune regulation [8]. Therefore, how to improve the activity and survival time of transplanted MSCs become urgent problems in DMED therapy.

Probucol has been used as anti-atherosclerotic for many years for its affection on anti-inflammatory and anti-oxidative. According to these pharmacological activities, probucol has been used in the treatment of DMED $[9,10]$. Our previous study found that probucol can improve erectile function in diabetic rats, which may be related to activation of Nrf2 pathway [11].

Many studies show that Nrf2 plays a protective role in many pathological processes, including DMED [11, 12]. Nrf2 is a basic leucine zipper REDOX sensitive transcription factor that can regulate the REDOX state of cells when exposed to harmful stimuli [13]. Under basic condition, Nrf2 locates in the cytoplasm with its anchor inhibitors Kelch sample ECH associate protein 1 (Keap1) [14]. However, under oxidative stress, Nrf2 disintegrates from Keap1, relocates to the nucleus, and regulates downstream oxidation resistance genes. The activation of Nrf2 was found to have anti-apoptotic effect [15]. In addition, recent studies have shown that Nrf2 can regulate the formation of autophagy, further promoting its protective effect $[16,17]$.

Based on the probucol's characteristics of antioxidation, anti-apoptosis, and upregulation of protective autophagy, we attempted to give diabetic rat MSCs combined with oral probucol in order to determine its effect on the treatment of DMED and further explore the possible mechanism.

\section{Methods}

\section{Experimental animals}

A total of forty-eight 10-week-old Sprague-Dawley male rats weighing 260-280 g were purchased from the Animal Center of Shandong University. Animal care and treatment were approved by the Animal Care and Use Committee of Shandong University (Jinan, China). Rats were maintained in specific pathogen-free environment around $23 \pm 1{ }^{\circ} \mathrm{C}$ with a 12-h light-dark cycle and supplied food and water ad libitum. All animals were adapted to the new environment for 1 week before the experiment. To establish diabetes, thirty-six rats were fasted for $12 \mathrm{~h}$, then received a single intraperitoneal injection of $60 \mathrm{mg} / \mathrm{kg}$ streptozotocin (STZ, Sigma-Aldrich Chemical Co, St. Louis, MO). Twelve rats were administered vehicle only and were used as a sham group. Random blood glucose levels were monitored $72 \mathrm{~h}$ later after STZ or vehicle injection. Only those STZ-treated rats with random blood glucose concentrations consistently greater than $16.7 \mathrm{mmol} / \mathrm{L}$ were accepted as being 
diabetic. These rats were divided randomly into the following 3 groups: (1) probucol (Sigma-Aldrich, St. Louis, $\mathrm{MO}, 500 \mathrm{mg} / \mathrm{kg} /$ day) was administered daily after intracavernous injection of MSCs ( $\mathrm{P}+\mathrm{M}$ group, $n=12)$, (2) intracavernous injection of MSCs $\left(1 \times 10^{6}\right)$ in diabetic rats (M group, $n=12$ ), and (3) the diabetic group (DM group, $n=12$ ). Initial and final blood glucose levels and body weight of all rats were recorded.

\section{Isolation and cultivation of MSCs}

Isolation and cultivation of bone marrow-derived stem cells were performed according to previous descriptions [18]. Typically, male Sprague-Dawley rats $(80-120 \mathrm{~g})$ were killed unconsciously; bone marrow tissues were washed with PBS until the bloodiness was eluted. After digestion with collagenase I and centrifugation, supernatant was discarded, and the residual cells were suspended in DMEM then cultured in humidified atmosphere and $5 \% \mathrm{CO}_{2}$ at $37^{\circ} \mathrm{C}$ for $48 \mathrm{~h}$. Cells were passaged when they reached about $90 \%$ confluence, and passage 3 cells were used in vivo and in vitro experiments. To verify the cellular identity of cells, fluorescence-activated cell sorting was employed via the usage of CD90, CD29, CD34, and CD45 markers.

\section{The label, intracavernous injection, and observation of MSCs}

Labeling of MSCs using Cm-Dil is performed under protocol. After anesthesia, the prepuce was rolled up to expose the penis; the needle was inserted by $3-4 \mathrm{~mm}$ under the microscope. MSCs $\left(1.0 \times 10^{6}\right.$ cells in fresh PBS $100 \mu \mathrm{L}$ ) or vehicle (fresh PBS $100 \mu \mathrm{L}$ only) was injected into the middle of the left corpus cavernosum. An elastic band was placed at the base of the penis immediately before MSC injection and was removed $3 \mathrm{~min}$ after the injection. Tissue was collected at 3 days, 1 week, and 2 weeks after transplantation, and frozen sections were analyzed by fluorescent microscope.

\section{Western blotting}

Protein extraction and Western blotting were performed as previously described [19]. Tissues and cells were lysed with RIPA buffer containing protease inhibitor cocktail, and the protein concentrations of tissue lysates and cell lysates were determined by BCA assay. Samples containing $20 \mu \mathrm{g}$ of protein were subjected to sodium dodecyl sulfate polyacrylamide gel electrophoresis and transferred to a polyvinylidene fluoride membrane. The membrane was blocked with $5 \%$ skim milk and incubated at $4{ }^{\circ} \mathrm{C}$ overnight with primary antibodies against LC3 (1: 3000, Abcam), p62 (1:1000, Cell Signaling Technology), Nrf2 (1:1000, Cell Signaling Technology), HO-1 (1:1000, Abcam), Beclin1 (1:1000 Cell Signaling Technology), Bcl-2 (1:1000,Cell Signaling Technology), Bax (1:1000,
Abcam), Caspase3 (1:1000, Cell Signaling Technology), and Cleaved-Caspase3 (1:2000; Cell Signaling Technology). After hybridization of secondary antibodies, the result was visualized with an LAS3000 Image Analyzer (Fujifilm, Tokyo, Japan) and data were analyzed using Multigauge software (Fujifilm).

\section{Immunofluorescence staining}

Penile tissue was fixed in fresh $4 \%$ paraformaldehyde and then immersed in $30 \%$ sucrose in PBS overnight at $4{ }^{\circ} \mathrm{C}$. The fixed tissues were cryoembedded in optimal cutting temperature compound (Sakura Finetek, Torrance, CA, USA) and cut into 5- $\mu \mathrm{m}$ sections before mounting on slides. After permeabilization and blocking, the slides were incubated with primary antibodies, including rabbit anti- $\alpha$-smooth muscle actin $(\alpha-S M A, 1$ : 1000; Abcam) and rabbit anti-von Willebrand factor (vWF, 1:2000; Abcam) at $4{ }^{\circ} \mathrm{C}$ overnight. At room temperature, the sections were rinsed and incubated with Alexa Fluor-594-conjugated secondary antibodies (Invitrogen, Carlsbad, CA, USA). Nuclei were stained by 4',6-diamidino-2-phenylindole (DAPI, Invitrogen) for 5 min. Slides were visualized under a fluorescence microscope (Leica, Heidelberg, Germany).

\section{Masson's trichrome stain}

Masson's trichrome stain was used to evaluate the smooth muscle cell and collagen fibril expression in cavernous tissue. Three-micrometer sections of formalinfixed, paraffin-embedded tissues were deparaffinized in xylene (3 washes for 3 min each) and hydrated in graded ethanol to distilled water. The slides were then stained with Masson's trichrome stain kit (Dako Sciences, Glostrup, Denmark), followed by dehydration in graded ethanol to xylene.

\section{Determination of cell viability using CCK8}

The CCK-8 assay was used to measure MSC cell viability. MSCs (1000/well) were seeded in 96-well plates overnight. Cells were incubated with different concentrations $(100,150,200$, and $250 \mu \mathrm{M})$ of $\mathrm{H}_{2} \mathrm{O}_{2}$ for $12 \mathrm{~h}$; normal culture media were used for the control group. Then, cells were first co-cultured with probucol (50, 100 , and $150 \mu \mathrm{M})$ for $12 \mathrm{~h}$ and then exposed to $\mathrm{H}_{2} \mathrm{O}_{2}$ $(250 \mu \mathrm{M})$ for $12 \mathrm{~h}$. Normal culture media were used for the control group. At the prespecified time points, $10 \mu \mathrm{L}$ of CCK-8 solution (DOJINDO, Kumamoto, Japan) was added to the cells. After incubation for another $4 \mathrm{~h}$, the optical density (OD) values were determined at $450 \mathrm{~nm}$ using a microplate reader (BioTek, Winooski, VT, USA). Each group was tested in triplicate in three replicates. 


\section{Erectile function evaluation}

Measurements of maximal intracavernous pressure ( $\max$ $\mathrm{ICP}$ ) and the ratio of max $\mathrm{ICP} /$ mean systemic arterial pressure (MAP) were used to assess erectile function. After treatment, rats were anesthetized with $5 \%$ sodium pentobarbital. PE-50 (Intramedic; Becton Dickinson \& Co., Sparks, MD) tubes were inserted into the left carotid artery of each rat to detect continuous measurement of MAP. A 26-gauge needle filled with heparin $(250 \mathrm{U} / \mathrm{mL})$ was inserted into the cavernous body of the penis to detect ICP. After finding and dissociating cavernous nerve $(\mathrm{CN})$, an electrical stimulus with a frequency of $15 \mathrm{~Hz}$ and a pulse width of $5 \mathrm{~ms}$ was used to stimulate 60s. ICP and MAP were measured continuously by a BL-420V pressure transducer system (AD instrument).

\section{Autophagic flux measurements}

To detect autophagic flux, RFP-GFP-LC3 reporter plasmid $(1 \mu \mathrm{L} / \mathrm{mL}$, Addgene, Cambridge, MA, USA) was transfected into MSCs using lipofectamine 2000 (Invitrogen, 11668-019) according to the manufacturer's instructions. Then, the transfected cells were grouped into 3 groups: the sham group, $\mathrm{H}_{2} \mathrm{O}_{2}$ group $(200 \mu \mathrm{M})$, and probucol group $(150 \mu \mathrm{M})$. The cell images were obtained using Olympus FV1000 laser scanning confocal microscopy (Olympus, Tokyo, Japan).

\section{ROS assay}

Cellular ROS levels were measured using cell permeable probe 5-(and-6)-chloromethyl-2',7'-dichlorodihydrofluorescein diacetate (CM-H2DCFDA, Thermo Fisher, Waltham, MA, USA). Cells were loaded with $10 \mu \mathrm{M}$ H2DCFDA in DMEM (phenol red-free) for $1 \mathrm{~h}$. After washing cells twice with DMEM, fluorescence was measured with an Envision 2104 Multilabel reader (Perkin Elmer, Waltham, MA, USA).

\section{Statistical analysis}

All experiments were repeated at least three times, and all data were presented as means \pm SD. Statistical significance was analyzed by the SPSS version 22.0 software (SPSS, IL, USA). Differences between two groups were assessed using the Student $t$ test, and between multiple groups using one-way ANOVA. Values of $P<0.05$ were considered significant.

\section{Results}

Probucol and MSCs had no effect on body weight and blood glucose level in diabetic rats

The body weights and blood glucose levels are shown in Table 1. Compared with the sham group, the diabetic rats showed significantly higher blood glucose levels but significantly lower body weights before the experiment $(P<0.01)$. Probucol or MSC treatment or combination of probucol and MSCs did not improve the change in blood glucose level. The body weight increased for probucol and MSC intervention $(P<0.05)$.

\section{Identification of MSCs and the survival of MSCs}

To characterize the MSCs used in this study, we analyzed the expression of cell surface antigens, and as shown in Fig. 1a, the cells expressed markers of MSCs including CD29 and CD90, but not the hematopoietic or endothelial markers CD34 and CD45. Furthermore, we used frozen sections to detect the survival of the MSCs. As shown in Fig. 1b, after 3 days post-transplantation, more DiIpositive cells (red) were observed in the $P+M$ group, compared with the M group, indicating that MSC survival was significantly improved due to the application of probucol. At 1 week, a large number of MSCs survived in the $\mathrm{P}+\mathrm{M}$ group, while very few cells were observed in the $\mathrm{M}$ group. At 2 weeks, cells remained retaining in the $\mathrm{P}+\mathrm{M}$ group while no cells could be observed in the $\mathrm{M}$ group.

\section{Probucol combined with MSCs improved erectile function of diabetic rats}

We used maximum ICP and ICP to MAP ratios to measure changes in erectile function in rats with different interventions. As shown in Fig. 1b, rats in the DM group exerted significant decreases in both maximum ICP and ICP to MAP ratios compared to the normal rats $(P<0.01)$. MSC treatment partially ameliorated erectile dysfunction compared with diabetic rats $(P<0.01)$ while rats treated with probucol displayed significantly higher function compared with MSC-injected rats $(P<0.01)$.

Table 1 Comparisons of body weight and blood glucose in experimental animals

\begin{tabular}{llllll}
\hline & & Sham group & DM group & M group & P + M group \\
\hline Initial & Body weight (g) & $293.4 \pm 21.78$ & $305.7 \pm 20.48$ & $306.5 \pm 21.05$ & $305.9 \pm 22.43$ \\
& Glucose (mmol/L) & $6.18 \pm 1.97$ & $20.87 \pm 1.03$ & $20.69 \pm 1.43$ & $21.09 \pm 1.13$ \\
4 weeks & Body weight (g) & $414.3 \pm 32.76$ & $303.7 \pm 14.38^{* *}$ & $343.4 \pm 17.88^{\#}$ & $356.9 \pm 18.51^{\#}$ \\
& Glucose (mmol/L) & $6.26 \pm 1.87$ & $21.58 \pm 2.59^{* *}$ & $21.39 \pm 1.98^{* *}$ & $21.69 \pm 2.81^{* *}$ \\
\hline
\end{tabular}

Data are expressed as mean \pm standard deviation from $n=6$ per group. DM group, diabetes mellitus group; M group, diabetic rats were treated by intracavernous injection of MSCs and gavage with normal saline; $P+M$, diabetic rats were treated by intracavernous injection of MSCs and gavage with probucol. ${ }^{*} P<0.05$ and

${ }^{* *} P<0.01$ indicate significant difference compared with the sham group. ${ }^{\#} P<0.05$ indicates significant difference compared with the DM group 


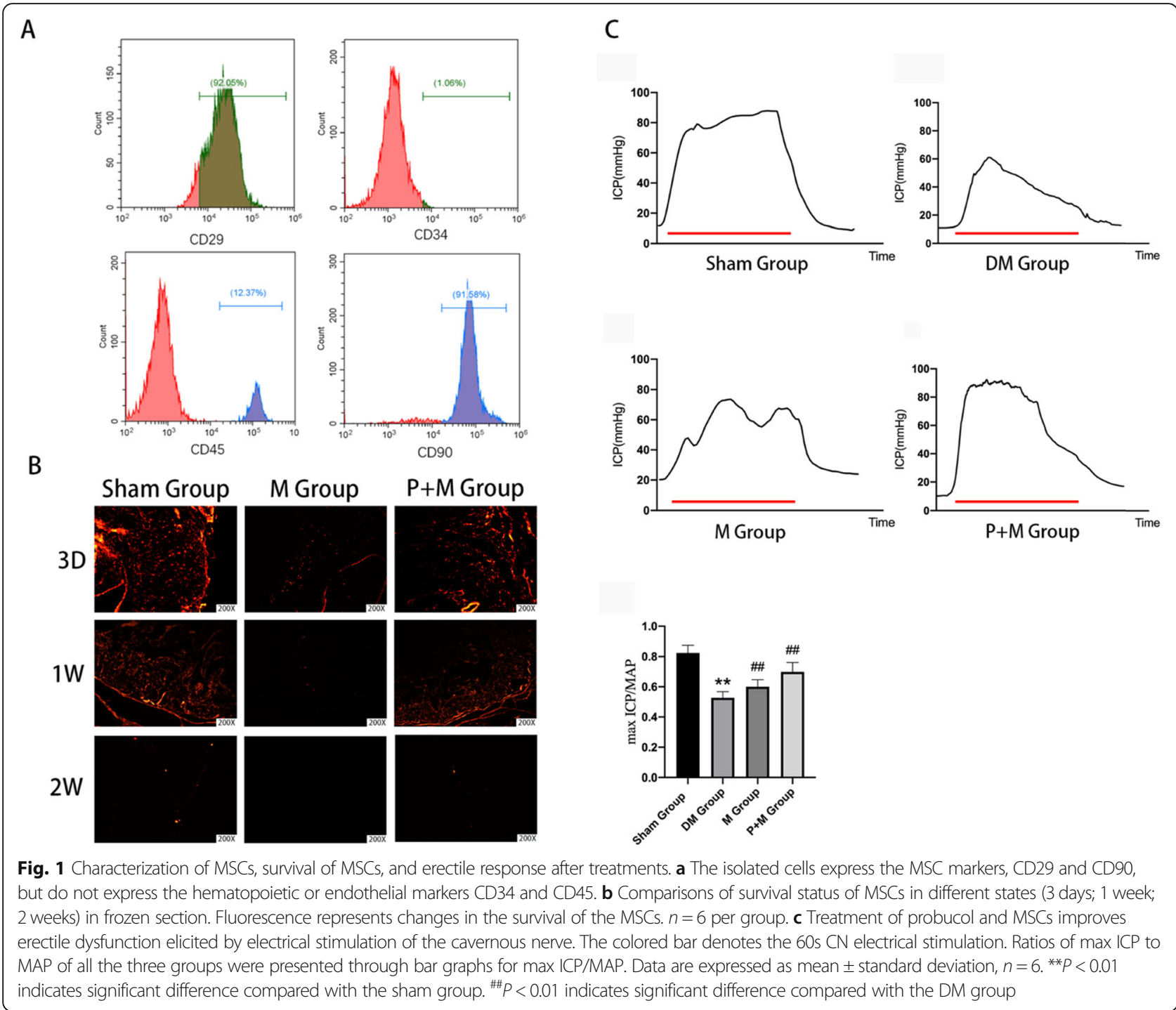

\section{Probucol combined with MSCs improved endothelial cell function}

Immunofluorescence staining analysis of $\alpha$-SMA expression showed a significant decrease of smooth muscle content in diabetic rats (Fig. 2c). The corpus cavernosum was evaluated for the smooth muscle/collagen ratios on slides stained with Masson's trichrome. As shown in Fig. 2a, both MSC and probucol treatments restored the smooth muscle content. The smooth muscle/collagen ratio in different groups is shown in Fig. 2e. Surprisingly, probucol treatment displayed a better recovery with higher smooth muscle density compared to the DM Group $(P<0.01)$. The von Willebrand factor (vWF)-positive area of the endothelium content in the DM group was significantly lower than in the sham group (Fig. 2b). After IC injection of MSCs, the endothelium content was increased to some degree. After probucol treatment, the endothelium in cavernous tissue was better restored compared with MSC-injected rats.
Probucol activated Nrf2/HO-1 antioxidant stress pathway In Fig. 2d, Western blotting results showed that diabetes mellitus caused significant decreased expression of Nrf2 and HO-1 in the corpus cavernosum $(P<0.01)$. Rats in the $\mathrm{P}+\mathrm{M}$ group showed a significant recovery of Nrf2 $(P<0.05)$ and HO-1 expression $(P<0.01)$ in 1 week. Obviously, after 2 weeks of treatment, probucol's intervention increased more Nrf2 and HO-1 expression compared with the DM group $(P<0.01)$.

Probucol reduced the level of ROS, increased cell viability, and activated Nrf2/HO-1 antioxidant stress pathway in $\mathrm{H}_{2} \mathrm{O}_{2}$-treated MSCs

To investigate the effect of probucol on MSCs under oxidative stress, we used CCK8 to detect the survival of MSCs under different conditions. In Fig. 3a, CCK8 results indicated that $\mathrm{H}_{2} \mathrm{O}_{2}$ induced a dose-dependent 


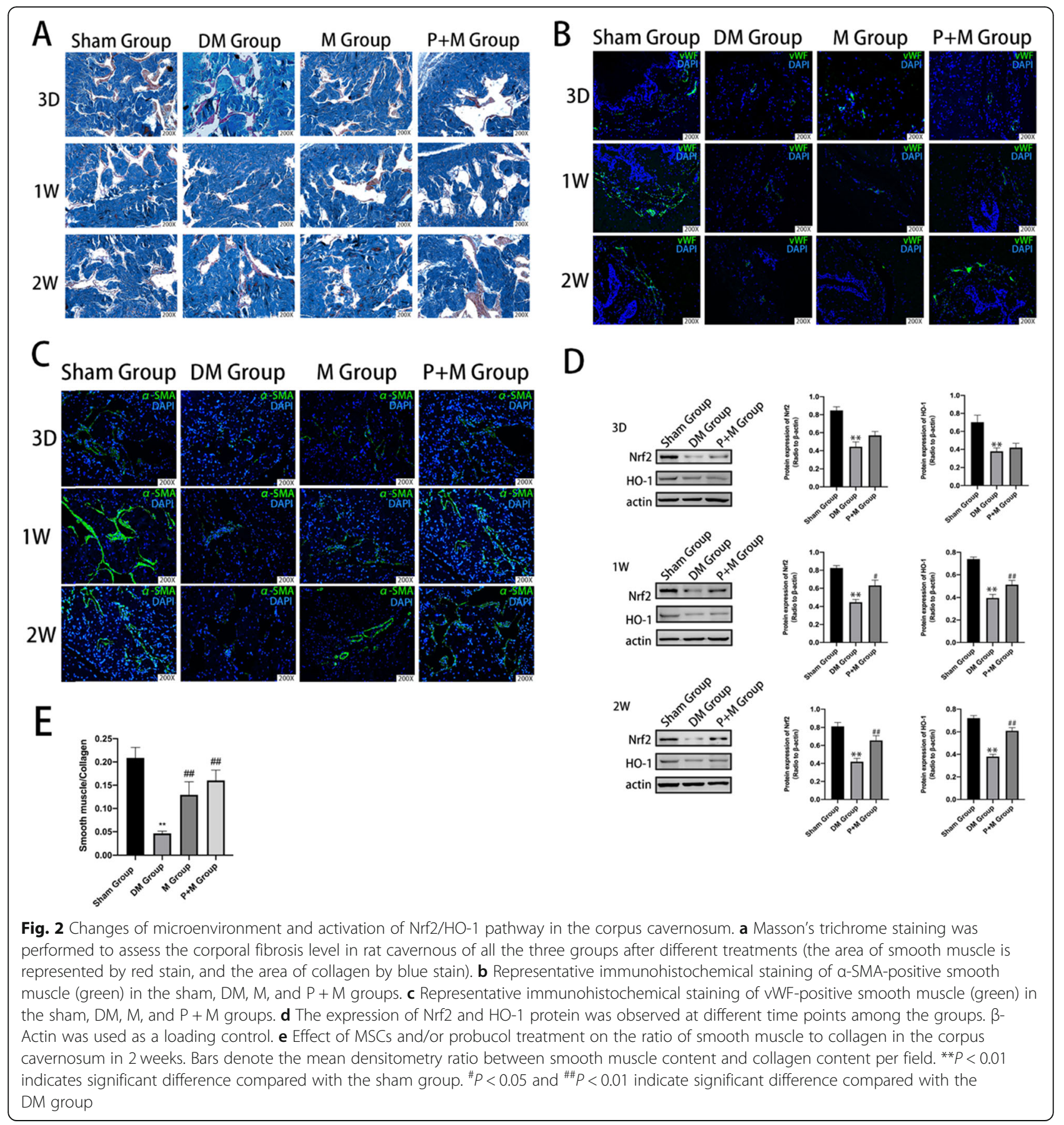

decrease of cell viability. MSC cell viability was reduced to $50 \%$ at $12 \mathrm{~h}$ by $200 \mu \mathrm{M} \mathrm{H}_{2} \mathrm{O}_{2}$, a concentration below its IC50 value $(250 \mu \mathrm{M})$, which was selected as a standard concentration for subsequent experiments. As shown in Fig. 3c, after exposure to $\mathrm{H}_{2} \mathrm{O}_{2}$, ROS production in MSCs increased. Compared to the $\mathrm{H}_{2} \mathrm{O}_{2}$ group, cell viability was significantly increased (Fig. $3 \mathrm{~b}$ ), and ROS production was dramatically decreased in the $\mathrm{H}_{2} \mathrm{O}_{2}$ + probucol $(150 \mu \mathrm{M})$ group in a dose-dependent manner (Fig. 3c).
Probucol decreases apoptosis in MSCs

Figure 4a suggests that under $\mathrm{H}_{2} \mathrm{O}_{2}$-induced oxidative stress, Nrf2 and HO-1 expression were significantly decreased $(P<0.01)$, while probucol could significantly upregulate Nrf2 and HO-1 expression in a dose-dependent manner, reaching a peak at $150 \mu \mathrm{M}(P<0.01)$. Besides, increased ratio of pro-apoptotic Bax to anti-apoptotic Bcl-2 induces mitochondrial cytochrome $\mathrm{C}$ release, a hallmark of apoptosis. We used Western blotting to detect the apoptosis of MSCs. The results in Fig. 4b 

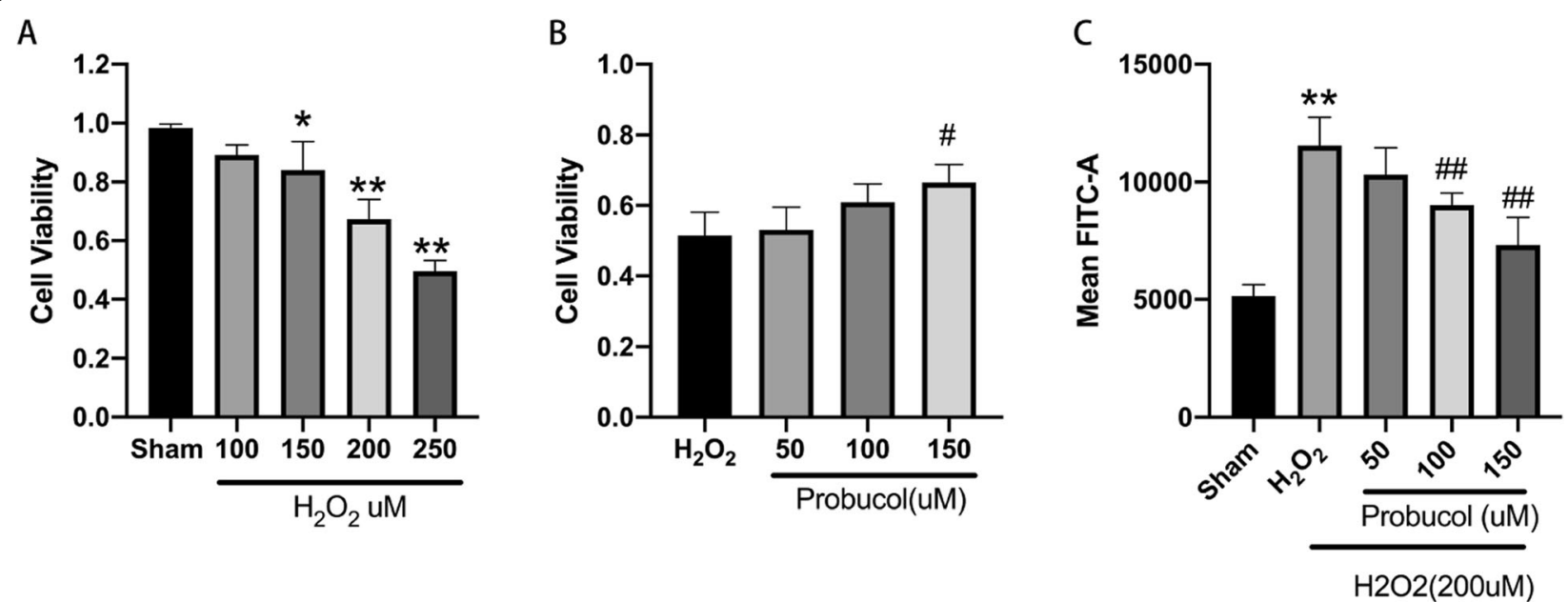

Fig. 3 Effect of $\mathrm{H}_{2} \mathrm{O}_{2}$ and probucol on MSC viability and ROS. a $\mathrm{H}_{2} \mathrm{O}_{2}$ treatment inhibits the viability of MSCs in a concentration-dependent manner. IC50 value was calculated using CCK8 assay in triplicates; sublC50 dose of $200 \mu \mathrm{M}$ was used for further experiences. b Probucol's intervention could reverse the injury of $\mathrm{H}_{2} \mathrm{O}_{2}$ to MSCs, and was statistically significant at the concentration of $150 \mu \mathrm{M}$. c Probucol reduces ROS levels in MSCs. Intracellular ROS in the normal group, the oxidative stress group, and the probucol group were measured by flow cytometry before histogram was made. ${ }^{*} P<0.05$ and ${ }^{* *} P<0.01$ indicate significant difference compared with the sham group. ${ }^{\#} P<0.05$ and ${ }^{\# \#} P<0.01$ indicate significant difference compared with the $\mathrm{H}_{2} \mathrm{O}_{2}$ group

exerted that $\mathrm{H}_{2} \mathrm{O}_{2}$ significantly increased the expression of Bax, Caspase3, and Cleaved-Caspase 3 and decreased the expression of $\mathrm{Bcl}-2$. The results indicated that $\mathrm{H}_{2} \mathrm{O}_{2}$ induced apoptosis of MSCs. The expression of Bax, Caspase3, and Cleaved-Caspase3 decreased, and the expression of Bcl-2 increased in a dose-dependent manner because of probucol's intervention at $150 \mu \mathrm{M}(P<0.01)$. These results suggested that probucol reduced $\mathrm{H}_{2} \mathrm{O}_{2}$-induced apoptosis.

\section{Probucol enhanced MSCs' protective autophagy}

Western blotting results in Fig. 5a showed that probucol could induce the expression of Beclin1 and LC3II in a dose-dependent manner, peaking at $150 \mu \mathrm{M}(P<0.01)$. At the same time, the expression of P62 decreased continuously $(P<0.01)$. In order to further determine whether probucol had a negative impact on autophagy and lysosome, we used RFP-GFP-LC3-transfected cells to mark autophagic vacuoles (yellow) and analyzed cell image by confocal microscope after intervention. As shown in Fig. 5b, after autophagy-lysosomal fusion, GFP was released from RFP-GFP-LC3 and degraded in lysosomes. Therefore, autolysosome would lead to the formation of fluorescence from yellow to red. In MSCs treated with probucol, autophagy lysosomes were observed, showing an increase in red spots. These results suggested that probucol could enhance the protective autophagy of MSCs by facilitating autophagosome-lysosome fusion.

\section{Discussion}

In recent years, stem cell transplantation therapy has been regarded as a promising weapon against ED.
Mesenchymal stem cells (MSCs) receive particular concerns as the result of their featured biological benefits, including abundance of autologous sources, ease of isolation, and expanding [20]. Meanwhile, many studies have shown that MSC transplantation had significant effect on a variety of diseases, including DMED [20-22]. In this study, we focused on the effect of the combination therapy and the impact of probucol on MSCs under oxidative stress. Our results indicated that the combined therapy could better improve the erectile function of diabetic rats, which might be related to the longer survival time of MSCs in the corpus cavernosum of diabetic rats. What is the mechanism? We thought it might be related to probucol-induced decrease in oxidative stress, increase in antioxidation and protective autophagy, and inhibition of apoptosis triggered by activation of the Nrf2 pathway.

Probucol diminished the oxidative stress level in the corpus cavernosum of diabetic rats. Nrf2-mediated antioxidant response to oxidant injuries has been detected in the corpus cavernosum from diabetic rats, suggesting that diabetic-related Nrf2 dysfunction increases vulnerability of the corpus cavernosum to oxidative stressinduced damage [11]. Nrf2 exerts its antioxidant activity by upregulating the expression of some effector enzymes, including HO-1 [23]. Our previous studies confirmed that activation of $\mathrm{Nrf} 2 / \mathrm{HO}-1$ pathway could reduce ROS production, which decreased the oxidative stress level of penile cavernous body in diabetic rats [11]. The results of current study showed the amounts of Nrf2 and HO-1 were significantly reduced in the penile cavernous body of diabetic rats, while probucol's 


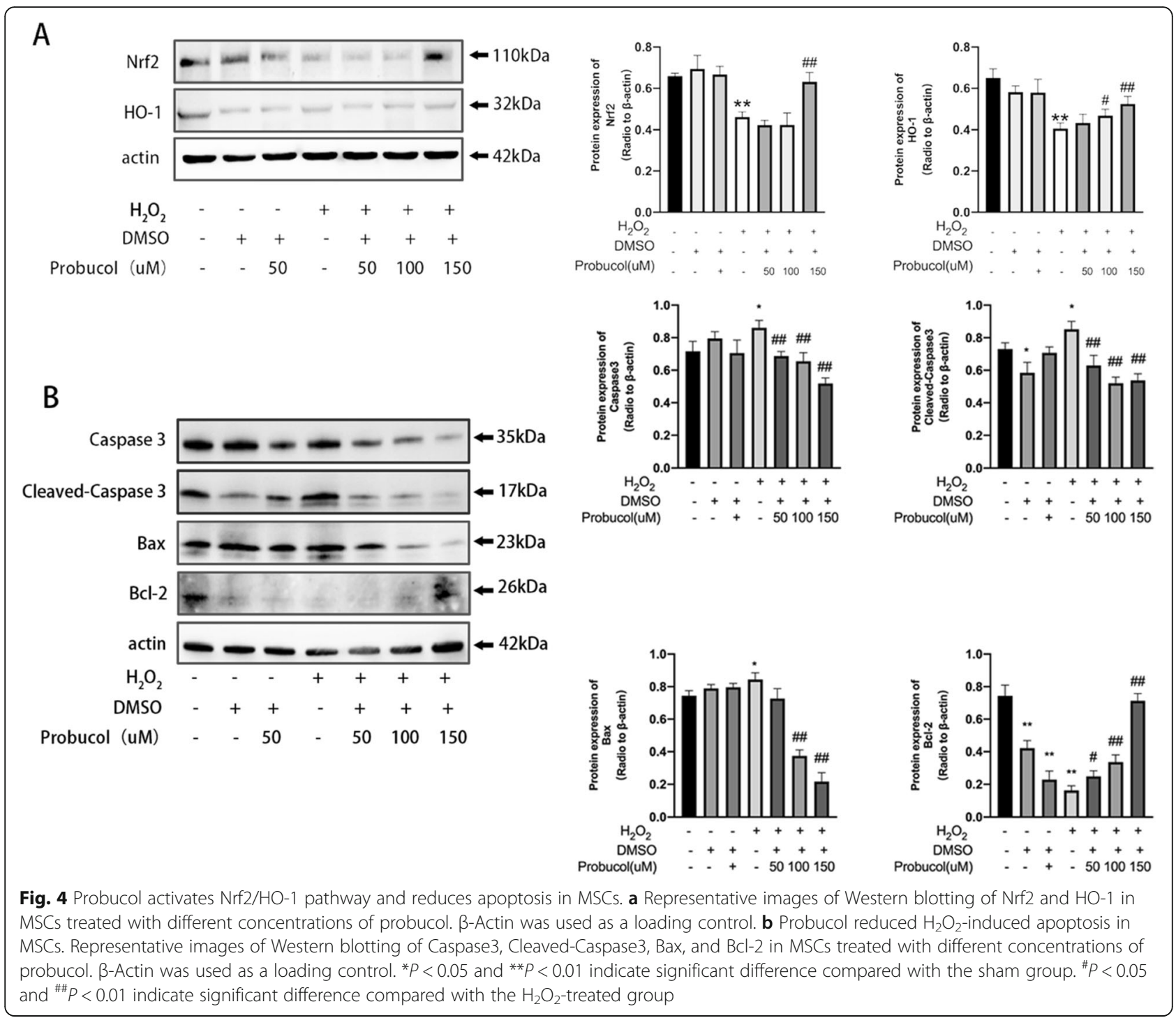

intervention reversed that and improved erectile function of diabetic rats. Therefore, it was proved that probucol could alleviate the hyperoxidative stress of penile cavernous body in diabetic rats and provide a good microenvironment for MSC transplantation.

Probucol enhanced self-antioxidant ability of MSCs through Nrf2. In in vitro experiments, we studied the effect of probucol on MSCs under oxidative stress. The secondary injury following $\mathrm{H}_{2} \mathrm{O}_{2}$ represents consecutive pathological processes including oxidative stress and apoptosis [24]. Oxidants and their dericatives generated by $\mathrm{H}_{2} \mathrm{O}_{2}$ enhance the generation of ROS and exhaust antioxidant defense enzymes. Level of expression of CCK8 showed probucol reverted the deleterious effect of $\mathrm{H}_{2} \mathrm{O}_{2}$ on MSC viability. Meanwhile, the activation of Nrf2 by probucol was dose-dependent and reached a peak at $150 \mu \mathrm{M}$. It is well documented that HO-1 is positively regulated by Nrf2 [25] which was confirmed by our results that the expression of $\mathrm{HO}-1$ increased with the increase of probucol in a dose-dependent manner. As an anti-oxidative stress protein, $\mathrm{HO}-1$ causes hemoglobin decomposition to form ferrous iron, carbon monoxide, and biliverdin, which resist the damage caused by oxidative stress [26]. Therefore, these data indicated that probucol enhanced MSCs' ability to resist oxidative stress by activating $\mathrm{Nrf} 2 / \mathrm{HO}-1$ pathway.

Probucol enhanced the protective autophagy of MSCs. It is not completely clear how autophagy is regulated by Nrf2, which do not get around P62. P62 is a lysosome protease substrate and has the dual binding sites of ubiquitin chains and LC3. P62 combines with LC3 through LC3 interaction area (LIR). Ubiquitin chains through UBA (related to ubiquitin) structure domain combine with LC3, so as to activate autophagy [27]. Thus, stimuli such as oxidative stress have been shown to induce autophagy and degradation of $\mathrm{P} 62$, and to reduce 


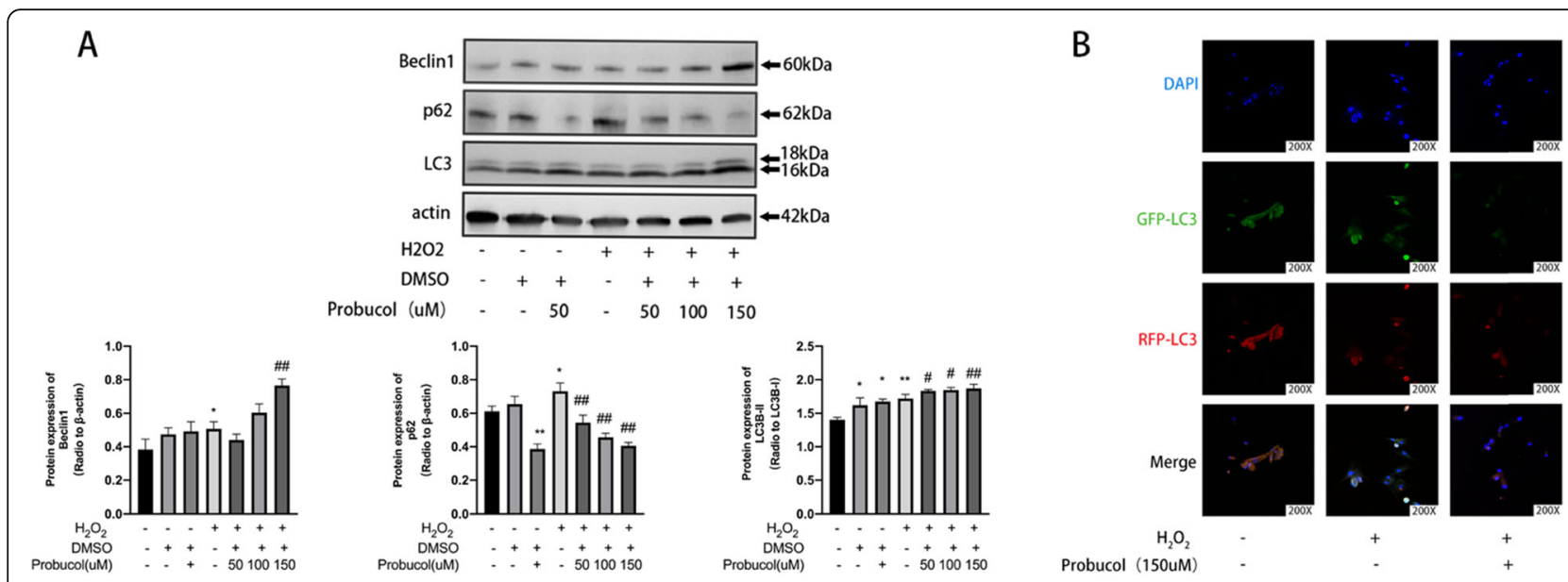

Fig. 5 Probucol activates autophagy of MSCs and does not block autophagy flux. a MSCs were exposed to probucol (0,50, 100, 150 $\mu \mathrm{M})$ for $12 \mathrm{~h}$ in the presence of $\mathrm{H}_{2} \mathrm{O}_{2}$. Western blotting analysis for expression levels of Beclin1, P62, and LC3B-II/I. The protein band intensities were normalized to $\beta$-actin, expressed relative to control. b MSCs were transfected with RFP-GFP-LC3. The cells were then incubated in the absence or presence of probucol $(150 \mu \mathrm{M})$ for $12 \mathrm{~h}$. Fluorescence microscopy images of MSCs were obtained by monitoring GFP-LC3 puncta (green

fluorescence), and the lysosomes were stained with RFP-LC3 (red fluorescence). Insets show an enlarged image of the merged field of interest

intracellular P62 levels [28]. Puissant and his colleagues suggested Nrf2 specificity to combine in P62 promoter of the antioxidant response element, promote the expression of $\mathrm{P} 62$, then combine with LC3, and activate autophagy [29]. Chunjuan Song and colleagues found that autophagy under oxidative stress was thought to be self-regulation of cells against harmful stimuli [30]. Our results suggest that probucol could increase Beclin1 and LC3-II levels and decrease the levels of P62 under oxidative stress. Moreover, autophagy flux suggested that probucol intervention did not inhibit autophagy flux, thus leading to autophagy accumulation. Double-labeled immunofluorescence analysis suggested that $\mathrm{H}_{2} \mathrm{O}_{2}$ inhibited the autophagy level of MSCs, resulting in GFP-LC3 accumulation in the cytoplasm. Probucol reversed this phenomenon by increasing expression of RFP-LC3, while activated autophagy and autophagy flux were not inhibited. These results suggested that probucol intervention enhanced MSCs' resistance to oxidative stress by increasing protective autophagy. Further studies are needed to clarify how Nrf2 regulates autophagy.

Probucol inhibited the apoptosis of MSCs under oxidative stress. Oxidative stress increases mitochondrial depolarization and $\mathrm{Ca}^{2+}$ entry, then induces mitochondrial release of cytochrome $\mathrm{C}$ into the cytosol and sequential activation of Caspase 3 and Caspase9, leading to apoptosis [31]. Previous experiments have shown that Caspase3 is a necessary pathway for the cascade of apoptotic proteases [32], and mitochondrial Bcl-2 family is the most important regulatory factor in the endogenous apoptotic pathway [33]. Apoptosis can be initiated through P53-induced activation of $\mathrm{Bax}$ in a caspase-dependent manner [34].
Our results showed that the expression of Bcl-2 was downregulated while $\mathrm{Bax}$ was upregulated in MSCs with $\mathrm{H}_{2} \mathrm{O}_{2}$ intervention. $\mathrm{H}_{2} \mathrm{O}_{2}$ intervention significantly upregulated the expression of apoptosis-related proteins Caspase3 and Cleaved-Caspase3, suggesting that $\mathrm{H}_{2} \mathrm{O}_{2}$ induced MSC apoptosis through mitochondrial Caspase3 pathway. Oral treatment with probucol reversed the above changes, leading to the upregulation of Bcl-2 and the downregulation of Bax, reducing downstream expression of Caspase 3 and CleavedCaspase3. These results suggested that $\mathrm{H}_{2} \mathrm{O}_{2}$ could break the balance between $\mathrm{Bcl}-2$ and $\mathrm{Bax}$ in the $\mathrm{Bcl}-2$ gene family, leading to a decrease in the Bcl-2/Bax ratio of MSCs under oxidative stress, thereby activating the mitochondrial Caspase3 pathway and promoting apoptosis. The possible mechanism was that probucol could activate Nrf2, thus upregulating the transcription of Bcl-2 gene. Furthermore, Bcl-2 mediated by Nrf2 lead to the decrease of Bax and apoptosis [35].

Furthermore, tissue structure was analyzed by Masson's trichrome staining and immunology staining with vascular endothelial marker (vWF) and vascular smooth muscle marker ( $\alpha$-SMA). Our previous studies have shown that probucol improves endothelial function and reduces fibrosis [36]. Our results shown that compared with MSCs alone, combination therapy increased the expression of vWF and $\alpha$-SMA. The above physiological processes worked together to restore the vascular endothelial function of penile cavernous body and reduce fibrosis. These changes provided the structural basis for the improvement of ED. The mechanism of structural change in the corpus cavernosum was not explored in this study. 


\section{Conclusion}

In conclusion, we demonstrate that probucol enhances the therapeutic efficiency of MSCs in the treatment of DMED by prolonging their survival time via Nrf2 pathway. These effects appear to be mediated through improved microenvironment of transplanted MSCs, by increasing their self-antioxidant ability, increasing protective autophagy, and diminishing apoptosis.

\section{Abbreviations}

MSCs: Mesenchymal stem cells; DMED: Diabetic mellitus-induced erectile dysfunction; IC: Intracavernosal; ED: Erectile dysfunction; DM: Diabetes mellitus; PDE5i: Phosphodiesterase type 5 inhibitors; ROS: Reactive oxygen species; Keap1: Kelch sample ECH associate protein 1; STZ: Streptozotocin; DMEM: Dulbecco's modified Eagle's medium; PBS: Phosphate buffer saline; aSMA: a-Smooth muscle actin; VWF: von Willebrand factor; OD: Optical density; CN: Cavernous nerve; ICP: Intracavernous pressure; MAP: Mean arterial blood pressure; LIR: LC3 interaction area

\section{Acknowledgements}

We are grateful to "Academic promotion plan" of Shandong first Medica University and engineering laboratory of urinary organ and functional reconstruction of Shandong province for their technical assistance in cell experiments.

\section{Authors' contributions}

Haoran Wang, Keqin Zhang, and Zheng Ruan are responsible for the animal experiments and related tests; Dingqi Sun, Hui Zhang, and Liangliang Hu have made great contributions to the molecular biological detection, especially the detection of Western blotting and autophagy flux; Guiting Lin, Shengtian Zhao, and Qiang Fu were responsible for the design of the experiment, assisted in writing the paper, and revised and approved the final version of the paper. All authors read and approved the final manuscript.

\section{Funding}

This work was supported by the National Natural Science Foundation of China (81873830); the Natural Science Foundation of Shandong Province (No. ZR2017BH036); the Key Research Development Program of Shandong Province (No. 2018GSF118142); the Shandong Provincial Key Research Program, China (2018GSF118083); and the Shandong Provincial Natural Science Foundation, China (ZR2019MH036).

\section{Availability of data and materials}

All data generated or analyzed during this study are included in the published article.

\section{Ethics approval and consent to participate}

The animal procedures in this study were approved by the Animal Care and Use Committee of Shandong University (Jinan, China).

\section{Consent for publication}

Not applicable.

\section{Competing interests}

The authors declare that they have no competing interests.

\section{Author details}

'Department of Urology, Shandong Provincial Hospital, Cheeloo College of Medicine, Shandong University, Jingwuweiqi Road 324\#, Jinan 250021, Shandong, People's Republic of China. ${ }^{2}$ Department of Urology, Shandong Provincial Hospital Affiliated to Shandong First Medical University, Jinan 250021, Shandong, People's Republic of China. ${ }^{3}$ Tai'an City Central Hospital, Tai'an 271000, People's Republic of China. ${ }^{4}$ Knuppe Molecular Urology Laboratory, Department of Urology, School of Medicine, University of California, San Francisco, CA, USA. ${ }^{5}$ Department of Urology, Shandong Zaozhuang Municipal Hospital, Zaozhuang 277000, People's Republic of China.
Received: 25 February 2020 Revised: 9 June 2020

Accepted: 23 June 2020 Published online: 21 July 2020

\section{References}

1. Irwin GM. Erectile dysfunction. Prim Care. 2019;46(2):249-55.

2. Kouidrat $Y$, et al. High prevalence of erectile dysfunction in diabetes: a systematic review and meta-analysis of 145 studies. Diabet Med. 2017;34(9): 1185-92.

3. Belba A, et al. Erectile dysfunction and diabetes: association with the impairment of lipid metabolism and oxidative stress. Clin Biochem. 2016; 49(1-2):70-8.

4. Moncada I, et al. Combination therapy for erectile dysfunction involving a PDE5 inhibitor and alprostadil. Int J Impot Res. 2018:30(5):203-8.

5. Yafi FA, Sharlip ID, Becher EF. Update on the safety of phosphodiesterase type 5 inhibitors for the treatment of erectile dysfunction. Sex Med Rev. 2018;6(2):242-52.

6. Matz EL, et al. Stem cell therapy for erectile dysfunction. Sex Med Rev. 2019; 7(2):321-8.

7. Yiou R, et al. Safety of intracavernous bone marrow-mononuclear cells for postradical prostatectomy erectile dysfunction: an open dose-escalation pilot study. Eur Urol. 2016;69(6):988-91.

8. Denu RA, Hematti P. Effects of oxidative stress on mesenchymal stem cell biology. Oxid Med Cell Longev. 2016;2016:2989076.

9. Xiao X, et al. Probucol protects rats from cardiac dysfunction induced by oxidative stress following cardiopulmonary resuscitation. Oxidative Med Cell Longevity. 2017:2017:1284804.

10. Zhang $\mathrm{M}$, et al. Probucol reverses homocysteine induced inflammatory monocytes differentiation and oxidative stress. Eur J Pharmacol. 2018:818: $67-73$.

11. $\mathrm{Hu} L \mathrm{~L}$, et al. Probucol improves erectile function via activation of Nrf2 and coordinates the HO-1 / DDAH / PPAR-gamma/ eNOS pathways in streptozotocin-induced diabetic rats. Biochem Biophys Res Commun. 2018; 507(1-4):9-14.

12. Bae WJ, et al. Antioxidant and antifibrotic effect of a herbal formulation in vitro and in the experimental andropause via Nrf2/HO-1 signaling pathway. Oxidative Med Cell Longevity. 2017;2017:6024839.

13. Tonelli C, Chio IIC, Tuveson DA. Transcriptional regulation by Nrf2 Antioxidants Redox Signaling. 2018;29(17):1727-45.

14. Kim, C.Y., et al., Parthenolide inhibits lipid accumulation via activation of Nrf2/Keap1 signaling during adipocyte differentiation. Food Sci Biotechnol. 2020;29(3):431-40

15. Méndez-García LA, et al. Curcumin induces p53-independent inactivation of Nrf2 during oxidative stress-induced apoptosis. Human Experimental Toxicol. 2019:38(8):951-61.

16. Li L, et al. ROS and autophagy: interactions and molecular regulatory mechanisms. Cell Mol Neurobiol. 2015:35(5):615-21.

17. Pajares $M$, et al. Transcription factor NFE2L2/NRF2 is a regulator of macroautophagy genes. Autophagy. 2016;12(10):1902-16.

18. Narita T, et al. The use of scaffold-free cell sheet technique to refine mesenchymal stromal cell-based therapy for heart failure. Mol Ther. 2013: 21(4):860-7.

19. Drechsel DA, Patel M. Differential contribution of the mitochondrial respiratory chain complexes to reactive oxygen species production by redox cycling agents implicated in parkinsonism. Toxicol Sci. 2009;112(2): 427-34.

20. Al Demour S, et al. Safety and potential therapeutic effect of two intracavernous autologous bone marrow derived mesenchymal stem cells injections in diabetic patients with erectile dysfunction: an open label phase I clinical trial. Urol Int. 2018:101(3):358-65.

21. Blau HM, Daley GQ. Stem cells in the treatment of disease. N Engl J Med. 2019;380(18):1748-60.

22. Mead B, et al. Stem cell treatment of degenerative eye disease. Stem Cell Res. 2015;14(3):243-57.

23. Chen $\mathrm{B}$, et al. <The role of Nrf2 in oxidative stress-induced endothelial injuries.pdf>. J. Endocrinol. 2015;225(3):83-99.

24. Zhang $\mathrm{H}$, et al. Gastrodin induced $\mathrm{HO}-1$ and Nrf2 up-regulation to alleviate $\mathrm{H} 2 \mathrm{O} 2$-induced oxidative stress in mouse liver sinusoidal endothelial cells through p38 MAPK phosphorylation. Brazilian J Med Biological Res. 2018; 51(10):e7439.

25. Chen $\mathrm{HH}$, et al. 4-Ketopinoresinol, a novel naturally occurring ARE activator, induces the $\mathrm{Nrf} / \mathrm{HO}-1$ axis and protects against oxidative stress-induced 
cell injury via activation of PI3K/AKT signaling. Free Radic Biol Med. 2012; 52(6):1054-66.

26. Lee JM, et al. Identification of the NF-E2-related factor-2-dependent genes conferring protection against oxidative stress in primary cortical astrocytes using oligonucleotide microarray analysis. J Biol Chem. 2003;278(14):1202938.

27. Noda NN, et al. Structural basis of target recognition by Atg8/LC3 during selective autophagy. Genes Cells. 2008;13(12):1211-8.

28. Liu WJ, et al. p62 links the autophagy pathway and the ubiqutinproteasome system upon ubiquitinated protein degradation. Cellular Molecul Biol Letters. 2016;21:29.

29. Puissant A, Fenouille N, Auberger P. When autophagy meets cancer through p62/SQSTM1. Am J Cancer Res. 2012;2(4):397-413.

30. Matsuda $Y$, et al. Intravenous infusion of bone marrow-derived mesenchymal stem cells reduces erectile dysfunction following cavernous nerve injury in rats. Sex Med. 2018;6(1):49-57.

31. Ovey IS, Naziroglu M. Homocysteine and cytosolic GSH depletion induce apoptosis and oxidative toxicity through cytosolic calcium overload in the hippocampus of aged mice: involvement of TRPM2 and TRPV1 channels. Neuroscience. 2015;284:225-33.

32. Choudhary GS, Al-Harbi S, Almasan A. Caspase-3 activation is a critical determinant of genotoxic stress-induced apoptosis. Methods Molecular Biol (Clifton, N.J.). 2015;1219:1-9.

33. Mortezaee $\mathrm{K}$, et al. Modulation of apoptosis by melatonin for improving cancer treatment efficiency: an updated review. Life Sci. 2019;228:228-41.

34. Xie Y-L, Zhang B, Jing L. MiR-125b blocks Bax/Cytochrome C/Caspase-3 apoptotic signaling pathway in rat models of cerebral ischemia-reperfusion injury by targeting p53. Neurol Research. 2018:40(10):828-37.

35. Niture SK, Jaiswal AK. Nrf2 protein up-regulates antiapoptotic protein BCl-2 and prevents cellular apoptosis. J Biol Chem. 2012;287(13):9873-86.

36. Zhang $\mathrm{KQ}$, et al. Probucol improves erectile function by restoring endothelial function and preventing cavernous fibrosis in streptozotocininduced diabetic rats. Urology. 2016;91:241 e9.

\section{Publisher's Note}

Springer Nature remains neutral with regard to jurisdictional claims in published maps and institutional affiliations.

Ready to submit your research? Choose BMC and benefit from:

- fast, convenient online submission

- thorough peer review by experienced researchers in your field

- rapid publication on acceptance

- support for research data, including large and complex data types

- gold Open Access which fosters wider collaboration and increased citations

- maximum visibility for your research: over $100 \mathrm{M}$ website views per year

At $\mathrm{BMC}$, research is always in progress.

Learn more biomedcentral.com/submissions 